\title{
OPERATORS WITH SINGULAR CONTINUOUS SPECTRUM, VI. GRAPH LAPLACIANS AND LAPLACE-BELTRAMI OPERATORS
}

\author{
BARRY SIMON \\ (Communicated by Palle E. T. Jorgensen)
}

\begin{abstract}
Examples are constructed of Laplace-Beltrami operators and graph Laplacians with singular continuous spectrum.
\end{abstract}

\section{INTRODUCTION}

In previous papers in this series $[6,3,5,2,8]$, we have explored the occurrence of singular continuous spectrum, particularly for Schrödinger operators and Jacobi matrices. In this note, we'll construct examples of graphs whose Laplacians and Riemannian manifolds whose Laplace-Beltrami operators have singular continuous spectrum. The idea will be to take models with considerable symmetry which reduce to Jacobi matrices or Schrödinger operators, and so reduce these two types of models to known cases.

The analysis is simple, almost trivial. However, since these are the first examples I know of graph Laplacians or Laplace-Beltrami operators with singular continuous spectrum, it seems worthwhile to make it explicit.

\section{Graph Laplacians}

By a graph $G=(V, I)$, we will mean a countable set of vertices $V$ and an incidence matrix $I_{i j}$ of zeros and ones obeying

(i) $I_{i j}=I_{j i} ; \quad I_{i i}=0$.

(ii) $N_{i}=\left\{j \mid I_{i j}=1\right\}$ is finite for each $i$. Let $r(i)=\#\left(N_{i}\right)=\sum_{j} I_{i j}$, the coordination number of $r$.

(iii) $\sup r(i)<\infty$.

The graph is called regular if $r(i)$ is a constant.

Given a graph $G$, we can define two bounded operators on $\ell_{2}(V)=\left\{\{u(n)\}_{n \in V} \mid\right.$ $\left.\sum_{n \in V}|u(n)|^{2}<\infty\right\}:$

$$
\begin{aligned}
& \left(L_{1} u\right)(i)=\sum_{j} I_{i j} u(j)=\sum_{j \in N_{i}} u(j), \\
& \left(L_{2} u\right)(i)=\sum_{j} I_{i j}(u(i)-u(j))=\sum_{j \in N_{i}}(u(i)-u(j)) .
\end{aligned}
$$

Received by the editors October 7, 1994

1991 Mathematics Subject Classification. Primary 47B39, 05C50, 35P05, 58C40.

This material is based upon work supported by the National Science Foundation under Grant No. DMS-9101715. The Government has certain rights in this material. 


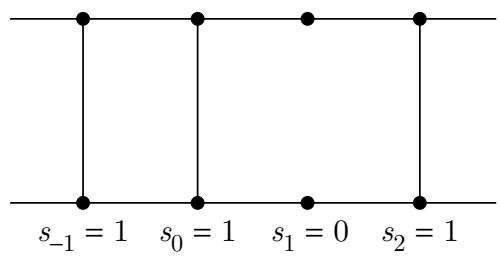

Figure 1. A ladder with missing rungs

$L_{1}$ and $L_{2}$ define quadratic forms by

$$
\begin{aligned}
\left(u, L_{1} u\right) & =\sum_{i j} I_{i j} \overline{u(i)} u(j), \\
\left(u, L_{2} u\right) & =\frac{1}{2} \sum_{i j} I_{i j}|u(i)-u(j)|^{2} .
\end{aligned}
$$

They are related by

$$
L_{2}=R-L_{1}
$$

where $(R u)(i)=r(i) u(i)$. In particular if $G$ is regular, $L_{1}$ and $L_{2}$ differ by a constant and sign and their spectral types are the same.

Our first example is a ladder with missing rungs. Let $V=\mathbb{Z} \times \mathbb{Z}_{2}=\{(n, \alpha) \mid$ $n \in \mathbb{Z}, \alpha=0$ or 1$\}$.

The incidence matrix will depend on the choice of a single sequence $s \in \mathbb{Z}_{2}^{\mathbb{Z}}$; that is, $s=\left\{s_{n}\right\}_{n \in \mathbb{Z}}$ with each $s_{n} 0$ or 1 . Given $s$, we define an incidence matrix so $I_{i j}=1$ if and only if

(1) $i=(n, \alpha), \quad j=(n \pm 1, \alpha)$ or

(2) $i=(n, 0), \quad j=(n, 1)$ or vice-versa where $s_{n}=1$.

Thus, as Figure 1 shows, the graph is a ladder with those rungs with $s_{n}=0$ missing.

Let $X=\mathbb{Z}_{2}^{\mathbb{Z}}$, a compact metric space in the product topology (and so complete). $s^{(m)} \rightarrow s^{(\infty)}$ if and only if $s_{n}^{(m)} \rightarrow s_{n}^{\infty}$ for each $n$. For each $s \in X$, we can define $L_{1}(s), L_{2}(s)$, the two Laplacians on $\ell_{2}(V)$. We say a set $S \subset X$ is generic if and only if $S$ is a dense $G_{\delta}$.

Theorem 2.1. For a generic $s \in X, L_{1}(s)$ has purely singular continuous spectrum and $L_{2}(s)$ has a spectrum with a non-zero purely singular component.

As a preliminary, we'll need:

Lemma 2.2. Fix $c \neq 0$. Given $s \in X$, let $J(s)$ be the Jacobi matrix on $\ell^{2}(\mathbb{Z})$ :

$$
(J(s) u)=u(n+1)+u(n-1)+c s_{n} u(n) .
$$

For each fixed $c,\{s \in X \mid J(s)$ has spectrum $[-2,2] \cup[c-2, c+2]$ and it is purely singular continuous $\}$ is a dense $G_{\delta}$.

Proof. Because a countable intersection of dense $G_{\delta}$ 's is again a dense $G_{\delta}$ (by the Baire category theorem), we need only show that each of

(i) $A_{1}=\{s \mid[-2,2] \subset \operatorname{spec}(J(s))$ and has no eigenvalues in $[-2,2]\}$,

(ii) $A_{2}=\{s \mid[c-2, c+2] \subset \operatorname{spec}(J(s))$ and has no eigenvalues in $[c-2, c+2]\}$,

(iii) $A_{3}=\{s \mid J(s)$ has no a.c. spectrum $\}$ 

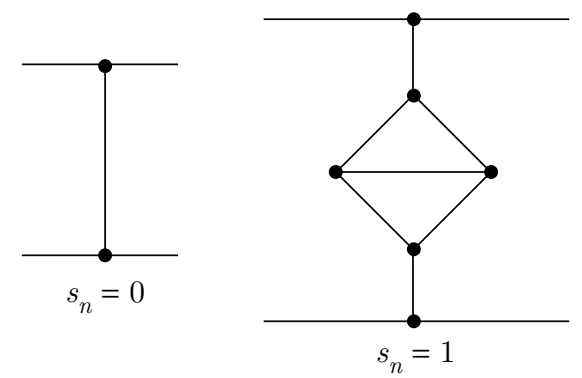

Figure 2. Rungs in the second example

is a dense $G_{\delta}$. Writing $J=J_{0}+V$ where $(V u)(n)=c s_{n}(u(n))$, and noting $\operatorname{spec}(V) \subset\{0, c\}$ and $\left\|J_{0}\right\| \equiv 2$, we have $\operatorname{spec}(J(s)) \subset[-2,2] \cup[c-2, c+2]$.

Each of the sets in (i)-(iii) is a $G_{\delta}$. That this is so for point and a.c. spectrum is a result of $[6,7]$. The argument that a given interval is in $\operatorname{spec}(A)$ on a $G_{\delta}$ is found in the appendix.

To see (i) is dense, note that $\left\{s \mid s_{n}=0\right.$ for $|n|$ large $\} \subset A_{1}$, and similarly, $\left\{s \mid s_{n}=1\right.$ for $|n|$ large $\} \subset A_{2}$. But those sets are dense.

For $A_{3}$, put any non-trivial product measure on $X$. By the result of Carmona, Klein, and Martinelli [1], for a.c. $s \in X$ with respect to this measure, $J(s)$ has pure point spectrum and, in particular, has a.c. spectrum. But the support of the product measure is all of $X$, so $A_{3}$ is dense.

Proof of Theorem 2.1. We'll use reflection symmetry about the middle of the ladder. Let $\mathcal{H}_{ \pm}=\left\{u \in \ell^{2}(V) \mid u(n, 1)= \pm u(n, 0)\right\}$ and let $W_{ \pm}: \ell^{2}(\mathbb{Z}) \rightarrow \mathcal{H}_{ \pm}$by

$$
\begin{aligned}
& \left(W_{ \pm} f\right)(n, 0)=f(n) / \sqrt{2}, \\
& \left(W_{ \pm} f\right)(n, 1)= \pm f(n) / \sqrt{2} .
\end{aligned}
$$

Then $\mathcal{H}_{ \pm}$is left invariant by both $L_{1}(s)$ and $L_{2}(s)$ and

$$
\begin{aligned}
& W_{ \pm}^{-1} L_{1}(s) W_{ \pm}=J( \pm s), \\
& W_{+}^{-1} L_{2}(s) W_{+}=2-J(0), \\
& W_{-}^{-1} L_{2}(s) W_{-}=2-J\left(-2 s_{n}\right) .
\end{aligned}
$$

The result now follows by the lemma.

Note that $L_{2}(s)$ always has an a.c. component of the spectrum since $J(0)$, the free Schrödinger Laplacian, has a.c. spectrum. This argument also shows that generically $\operatorname{spec}\left(L_{1}(s)\right)=[-3,3]$ and $\operatorname{spec}\left(L_{2}(s)\right) \equiv[0,6]$ with a.c. spectrum on $[0,4]$ and singular spectrum on $[2,6]$.

We were able to find a cheap trick to reduce the first example to the well-studied Jacobi case. That will not be true in the second example, and so we'll settle for a partial result. A complete analysis should be possible (I expect purely singular continuous spectrum for a generic $s$ ) but will require some effort to extend Carmona et al. [1] to this example.

Our purpose in this second example is to find a regular lattice whose Laplacian has singular spectrum; the coordination number in Figure 1 is sometimes 2 and sometimes 3. Our lattice will again depend on $s \in \mathbb{Z}_{2}^{\mathbb{Z}}$ and again be a basic ladder whose rungs are of the two types shown in Figure 2 if $s_{n}=0$ or $s_{n}=1$. 
The corresponding lattices are regular with coordination number 3 , so we'll only look at $L_{1}$, which we'll call $L(s)$. As before, the spaces $\mathcal{H}_{ \pm}$are invariant and we'll look only at the operator on $\mathcal{H}_{-}$(call the restriction of $L(s)$ to $\mathcal{H}_{-}, B(s)$ ). The space on which $B(s)$ acts is the pair of functions $u: \mathbb{Z} \rightarrow \mathbb{C}$ and $\varphi:\left\{n \mid s_{n} \equiv 1\right\} \equiv$ $Y(s) \rightarrow \mathbb{C}$ with $\sum_{n \in \mathbb{Z}}|u(n)|^{2}+\sum_{n \in Y(s)}|\varphi(n)|^{2}<\infty$ and with

$$
\begin{array}{rlrl}
(B(s) u)(n) & =u(n-1)+u(n+1)-u(n) \quad & \text { if } & s_{n}=0 \\
& =u(n+1)+u(n-1)+\varphi(n) \quad \text { if } \quad s_{n}=1, \\
(B(s) \varphi)(n) & =u(n) . & &
\end{array}
$$

We want to compute $\operatorname{spec}(B(s))$ for the two degenerate cases $s_{n} \equiv 0$ and $s_{n} \equiv 1$. If $s_{n} \equiv 0$, we get a Jacobi matrix whose spectrum is well known.

$B\left(s_{n} \equiv 0\right)$ has spectrum $[-3,1]$ with purely a.c. spectrum. For $s_{n} \equiv 1$, one looks at plane waves $u(n)=c e^{i k n}$, in which case $\varphi(n)=c E^{-1} e^{i k n}$ and $E$ obeys $E=2 \cos k+E^{-1}$, which leads to

$$
E=\cos k \pm \sqrt{1+\cos ^{2} k}
$$

It is not hard to see that these continuous eigenfunctions are complete and so

$$
\operatorname{spec}\left(B\left(s_{n} \equiv 1\right)\right)=[-1-\sqrt{2}, 1+\sqrt{2}] \text { and is purely absolutely continuous. }
$$

Theorem 2.3. For a generic $s \in X, B(s)$ contains $[-3,-1-\sqrt{2})$ in its spectrum and the spectrum is purely singular continuous there.

Proof. The spaces on which $B(s)$ act are $s$-dependent, but there is still a natural sense in which $s^{(n)} \rightarrow s^{(\infty)}$ means $\left(B\left(s^{(n)}\right)-z\right)^{-1} \rightarrow\left(B\left(s^{(\infty)}\right)-z\right)^{-1}$ strongly (apply to vectors of compact support) and this notion is such that the results of $[6,7]$ still apply. Let

$$
\begin{aligned}
& A_{1}=\{s \mid[-3,-1-\sqrt{2}) \subset \operatorname{spec}(B(s)) \text { and has no point eigenvalues there }\}, \\
& A_{2}=\{s \mid B(s) \text { has no a.c. spectrum on }[-3,-1-\sqrt{2})\} .
\end{aligned}
$$

Each is a $G_{\delta}$ by $[6,7]$.

We claim that if $s_{n} \equiv 0$ near infinity, $s \in A_{1}$; but if $s_{n} \equiv 1$ near infinity, then $s \in A_{2}$. This implies $A_{1}$ and $A_{2}$ are dense, so their intersection is a dense $G_{\delta}$ and Theorem 2.3 is proven.

The claim for $s_{n} \equiv 0$ near infinity follows by using plane waves near infinity as a Weyl sequence (to see that $[-3,1] \subset \operatorname{spec}(B(s))$ ) and then noting that eigenvalues must have compact support and then be zero by a simple argument. The claim for $s_{n} \equiv 1$ near infinity follows because $B(s)$ differs from $B\left(s_{n} \equiv 1\right.$ all $\left.n\right)$ by a finite rank operator, so $\operatorname{spec}(B(s))$ is thus discrete outside $[-1-\sqrt{2}, 1+\sqrt{2}]$.

\section{Some Laplace-Beltrami operators}

In this section, we'll find metrics on $\mathbb{R} \times S^{1}=M$ for which the Laplace-Beltrami operator has purely singular spectrum. Use coordinates $(x, \theta)$ on $M$ and consider metrics of the form $d x^{2}+f(x)^{2} d \theta$. We'll consider $1 \leq f(x) \leq 2$ and $f(x)$ is $C^{\infty}$ with the metric topology associated to $\left\{\sup _{|x| \leq N}\left\|\frac{d^{m} f}{d x^{m}}\right\|_{\infty}\right\}_{N=1,2, \ldots}^{m=0,1, \ldots}$. Denote by $X$ the set of such f's. Then: 
Theorem 3.1. For a generic $f$ in $X$, the Laplacian operator $H_{f}$ has spectrum $[0, \infty)$ and is purely singular continuous.

Proof. By a simple calculation (see, for example, [4]), under the decomposition $L^{2}\left(\mathbb{R} \times S^{1}\right) \cong \bigoplus_{n=-\infty}^{\infty} L^{2}(\mathbb{R})\left(\right.$ with $\left.g(z)=\sum g_{n}(x) e^{i n \theta}\right) H_{f} \cong \bigoplus H_{f ; n}$ and $H_{f ; n}$ is unitarily equivalent to the operator $L_{f ; n}$ on $L^{2}(\mathbb{R}, d x)$ with

where

$$
L_{f ; n}=-\frac{d^{2}}{d x^{2}}+V(x)+\frac{n^{2}}{f^{2}(x)} \equiv-\frac{d^{2}}{d x^{2}}+V_{n}(x)
$$

$$
V(x)=\frac{1}{4}\left(\frac{f^{\prime}}{f}\right)^{2}+\frac{1}{2}\left(\frac{f^{\prime}}{f}\right)^{\prime}
$$

We'll show for each $n$ that $L_{f ; n}$ generically has purely singular spectrum on $\left[\frac{n^{2}}{4}, \infty\right)$ and that implies the full result. If $f \equiv 2$ near infinity, $L_{f ; n}$ is $\frac{n^{2}}{4}$ plus a Schrödinger operator with the potential of compact support. This operator has no point spectrum in $\left[\frac{n^{2}}{4}, \infty\right)$. Since $L_{f ; n=0} \geq 0$ and $f \leq 2$, in general $L_{f ; n} \geq \frac{n^{2}}{4}$. Moreover, the functions $f \equiv 2$ near $\infty$ are dense in the topology on $X$. Thus, generically $L_{f ; n}$ has spectrum $\left[\frac{n^{2}}{4}, \infty\right)$ with no point spectrum. We'll need to show that generically there is no a.c. spectrum either.

Consider a metric of the form $f(x)=\alpha+\lambda \cos (k x)$ where $1<\alpha<2$ and $\lambda$ is small. Then

$$
V_{n}(x)=\frac{n^{2}}{\alpha^{2}}+\lambda\left[\frac{k^{2}}{2 \alpha}-\frac{n^{2}}{\alpha^{2}}\right] \cos (k x)+0\left(\lambda^{2}\right) .
$$

By the standard theory of periodic Schrödinger operators, for $\lambda$ small, $-\frac{d^{2}}{d x^{2}}+V_{n}(x)$ has a gap of order $\lambda$ about every $\frac{n^{2}}{\alpha^{2}}+\left(\frac{k}{2}\right)^{2}$. If $f(x) \equiv \alpha+\lambda \cos (k x)$ near infinity, $-\frac{d^{2}}{d x^{2}}+V_{n}(x)$ has only point spectrum in that interval. Thus, for any $E_{0} \in\left[\frac{n^{2}}{4}, \infty\right)$, we can find $\delta$ and a dense set of $f$ 's with no a.c. spectrum in $\left(E_{0}-\delta, E_{0}+\delta\right)$. By a compactness argument, we conclude that generically there is no a.c. spectrum. $\square$

Is it true that for any non-compact manifold, a generic metric leads to purely singular continuous spectrum?

\section{Appendix: $G_{\delta}$ 's And the SPECTRUm of Operators}

Recall that a regular space of operators is a family of selfadjoint operators indexed by $x \in X$ a complete metric space so that $x_{n} \rightarrow x$ in the metric topology implies that $A_{x_{n}} \rightarrow A_{x}$ in strong resolvent sense.

Our purpose here is to prove that

Theorem A.1. Let $[a, b]$ be a fixed closed interval and $X$ a regular space of operators. Then $\left\{x \mid[a, b] \subset \operatorname{spec}\left(A_{x}\right)\right\}$ is $a G_{\delta}$.

Let $\left\{\varphi_{n}\right\}_{n=1}^{\infty}$ be an orthonormal basis for the underlying Hilbert space, and let $d \mu_{n}(x)$ be the spectral measure defined by

$$
\left(\varphi_{n}, e^{-i t A} \varphi_{n}\right)=\int e^{-i t E} d \mu_{n}(E)
$$

and $d \mu=\sum_{n=1}^{\infty} 2^{-n} d \mu_{n}$. Then $\operatorname{spec}(A)=\operatorname{supp}(d \mu)$, so Theorem A.1 is a consequence of 
Lemma A.2. Let $M_{+, 1}^{R}$ denote the family of probability measures on $[-R, R] \subset \mathbb{R}$. Fix $[a, b] \subset[-R, R]$. Then $\left\{\mu \in M_{+, 1}^{R} \mid[a, b] \subset \operatorname{supp}(d \mu)\right\}$ is a $G_{\delta}$ in the topology of weak convergence.

Remark. Thus, if $S \subset M_{+, 1}^{R}$ is closed, $S \cap\{\mu \mid[a, b] \subset \operatorname{supp}(d \mu)\}$ is a $G_{\delta}$ in $S$.

Proof of Lemma A.2. Let $I_{n}$ be a counting of open intervals $I_{n} \subset[a, b]$ with rational endpoints. Let $f_{n}(x)=\operatorname{dist}\left(x, \mathbb{R} \backslash I_{n}\right)$. Then $[a, b] \subset \operatorname{supp} \mu$ if and only if for all $n$, $\int f_{n}(x) d \mu>0$, that is,

$$
\{\mu \mid[a, b] \subset \operatorname{supp}(d \mu)\}=\bigcap_{n=1}^{\infty}\left\{\mu \mid \int f_{n}(x) d \mu>0\right\}
$$

is clearly a $G_{\delta}$.

Remark. Using $\lim _{\epsilon \downarrow 0} \frac{1}{\pi} \int_{c}^{d} \operatorname{Im} F_{\mu}(x+i \epsilon) d x=\frac{1}{2} \mu((c, d))+\frac{1}{2} \mu([c, d])$ with $F_{\mu}(z)=$ $\int d \mu(x) /(x-z)$, it is easy to show that $\{\mu \mid[a, b] \cap \operatorname{supp} d \mu=\emptyset\}$ is also a $G_{\delta}$.

\section{REFERENCES}

[1] R. Carmona, A. Klein, and F. Martinelli, Anderson localization for Bernoulli and other singular potentials, Commun. Math. Phys. 108 (1987), 41-66. MR 88f:82027

[2] R. del Rio, S. Jitomirskaya, Y. Last, and B. Simon, Operators with singular continuous spectrum, IV. Hausdorff dimension and rank one perturbations, in preparation.

[3] R. del Rio, N. Makarov, and B. Simon, Operators with singular continuous spectrum, II. Rank one operators, Commun. Math. Phys. 165 (1994), 59-67. CMP 95:02

[4] V. Jaksic, S. Molchanov, and B. Simon, Eigenvalue asymptotics of the Neumann Laplacian of regions and manifolds with cusps, J. Funct. Anal. 106 (1992), 59-79. MR 93f:35165

[5] S. Jitomirskaya and B. Simon, Operators with singular continuous spectrum, III. Almost periodic Schrödinger operators, Commun. Math. Phys. 165 (1994), 201-205. CMP 95:02

[6] B. Simon, Operators with singular continuous spectrum, I. General operators, Ann. Math. 141 (1995), 131-145.

[7] $-L^{p}$ norms of the Borel transform and the decomposition of measures, Proc. Amer. Math. Soc. 123 (1995), 3749-3755.

[8] B. Simon and G. Stolz, Operators with singular continuous spectrum, V. Sparse potentials, Proc. Amer. Math. Soc. (to appear).

Division of Physics, Mathematics, and Astronomy, California Institute of Technology, 253-37, Pasadena, California 91125 